\title{
Intensities of quantum transitions in hexagonal nanotubes within the exciton spectral range
}

\author{
O.M. Makhanets, N.R. Tsiupak, O.M. Voitsekhivska \\ Chernivtsi National University, 2, Kotsiubynsky str., 58012 Chernivtsi, Ukraine, \\ Phone: +380 (3722) 44 816; e-mail:ktf@chnu.edu.ua
}

\begin{abstract}
The theory of exciton spectrum and intensities of interband quantum transitions in multi-shell hexagonal semiconductor nanotube is developed within the effective masses and rectangular potentials approximations using Bethe variational method. The obtained theoretical results well explain the experimental position of luminescence peak for $\mathrm{GaAs} / \mathrm{Al}_{\mathrm{x}} \mathrm{Ga}_{1-\mathrm{x}} \mathrm{As}$ nanotubes.
\end{abstract}

Keywords: hexagonal nanotube, quantum wire, exciton spectrum.

Manuscript received 26.01.12; revised version received 28.02.12; accepted for publication 27.03.12; published online 30.05.12.

\section{Introduction}

The intensive investigations of semiconductor quantum wires with radial heterostructure have been rapidly increased during the last decade. These nanostructures are the basic elements of light radiating diodes, detectors, transistors and light transformers of new generation.

On the one hand, the heterostructure perpendicular to the quantum wire axis can localize charge carriers inside the inner wire, decreasing the surface scattering [1]. On the other hand, it allows the guided variation of spectral characteristics inherent to quasi-particles (electrons, excitons, phonons) in nanostructure, depending on its geometrical parameters.

Using different semiconductor materials composing the radial heterostructure, the single (with one quantum well for the electron) [2, 3] and complicated (multi-well and multi-shell) $[4,5]$ hexagonal nanotubes are experimentally created. The single hexagonal nanotubes are already theoretically studied in details [6-8]. The authors developed the theory of exciton spectrum and exciton-phonon interaction, well explaining the experimental data qualitatively and quantitatively.

Recently, German scientists have been grown the complicated multi-shell hexagonal nanotubes by applying the method of molecular-beam epitaxy and using GaAs as well as $\mathrm{Al}_{\mathrm{x}} \mathrm{Ga}_{1-\mathrm{x}} \mathrm{As}$ semiconductors and investigated their luminescence spectra $[4,5]$. As far as we know, the consistent theory of exciton spectrum for these structures is still absent. However, even within the general considerations, it is clear that the complicated character of redistribution of probability for electron and hole location in the space of complicated nanotube with several quantum wells causes interesting peculiarities of the exciton spectrum. In particular, one can expect oscillations in intensities of interband quantum transitions.

In this paper, we propose one of the possible theoretical approaches to investigate the exciton spectrum in multi-shell hexagonal semiconductor nanotube. The theory is developed in the frames of models of effective masses and rectangular potentials for the quasi-particles using the Bethe variational method. The dependences of exciton spectrum parameters on nanostructure geometry have been investigated. The calculated magnitudes of exciton energies have been compared with the position of luminescence peak observed experimentally [5].

\section{Theory of electron and exciton spectra multi-shell hexagonal semiconductor nanotubes}

The experimentally grown nanostructure [5] - the multishell hexagonal nanotube is theoretically studied. It consists of hexagonal semiconductor quantum wire 
("0"), thin barrier-shell (" 1 ") and nanotube ("2") embedded into the outer medium ("3"). The transversal cross-section of the nanostructure is shown in Fig. 1.

The small differences of lattice and dielectric constants of nanostructures composing parts allow us to use the approximations of effective masses $\left(\mu^{(e, h)}\right)$ and rectangular potentials $\left(U^{(e, h)}\right)$ in order to calculate the electron and hole energy spectra. Their respective magnitudes are fixed as

$$
\begin{aligned}
\mu^{(e, h)}(\vec{r}) & =\left\{\begin{array}{l}
\mu_{0}^{(e, h)} \\
\mu_{1}^{(e, h)}
\end{array},\right. \\
U^{(e, h)}(\vec{r}) & = \begin{cases}0, & \text { media "0", "2" } \\
U_{0}^{(e, h)}, & \text { media "1", "3" }\end{cases}
\end{aligned}
$$

So that, to study the exciton spectrum we, first of all, obtain the electron and hole energy spectra and wave functions solving the stationary Schroedinger equation

$$
\hat{H}^{(e, h)} \Psi^{(e, h)}(\rho, \varphi, z)=E^{(e, h)} \Psi^{(e, h)}(\rho, \varphi, z)
$$

with Hamiltonian of uncoupling quasi-particles in the cylindrical coordinate system

$$
\begin{aligned}
& \hat{H}^{(e, h)}=-\frac{\hbar^{2}}{2}\left(\vec{\nabla}_{\rho, \varphi}^{(e, h)} \frac{1}{\mu^{(e, h)}(\rho, \varphi)} \vec{\nabla}_{\rho, \varphi}^{(e, h)}+\frac{1}{\mu^{(e, h)}(\rho, \varphi)} \frac{\partial^{2}}{\partial z^{2}}\right)(3) \\
& +U^{(e, h)}(\rho, \varphi) .
\end{aligned}
$$

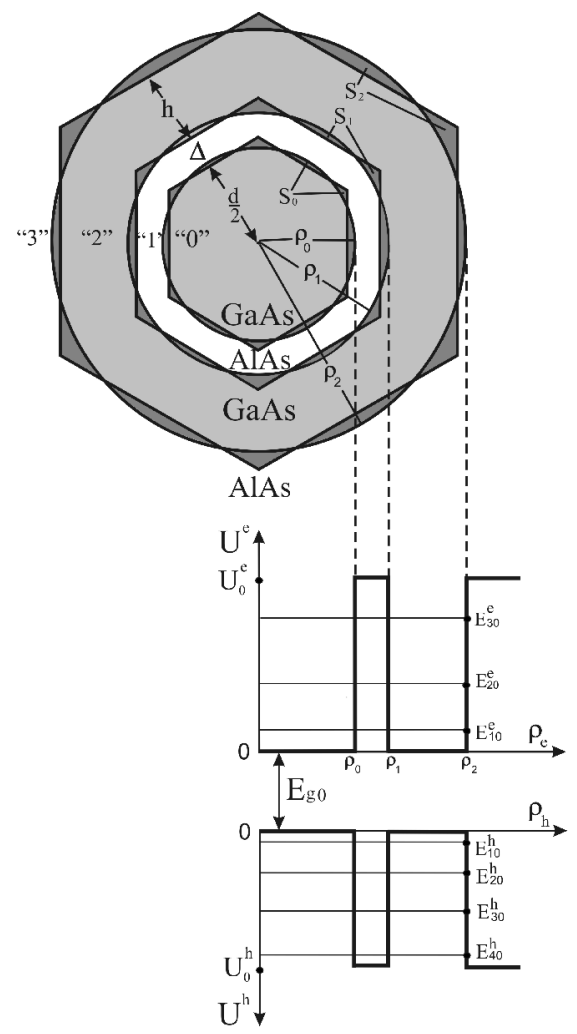

Fig. 1. Transversal cross-section of the nanostructure and its energy scheme.
It is clear that the effective masses $\left(\mu^{(e, h)}\right)$ and potential energies $\left(U^{(e, h)}\right)$ as functions of $\rho, \varphi$ variables have hexagonal symmetry in the plane perpendicular to the nanotube axis. Thus, the variables are not separated, and the equations (2) can not be solved exactly. The approximated solution is found within the Bethe variational method. In the Hamiltonian (3), the main term is introduced. In it, $\mu^{(e, h)}$ and $U^{(e, h)}$ magnitudes are the functions of radial variable $(\rho)$. I.e., the hexagons are replaced by the circles of respective radii: $\rho_{0}, \quad \rho_{1}=\rho_{0}+\Delta, \quad \rho_{2}=\rho_{0}+\Delta+h$. The differences between the respective masses: $\mu^{(e, h)}(\rho, \varphi)$ and $\mu^{(e, h)}(\rho)$, and potentials: $U^{(e, h)}(\rho, \varphi)$ and $U^{(e, h)}(\rho)$, arising as a result of approximation, are taken into account in the Hamiltonian as perturbation. Herein, the radius of the smallest circle $\left(\rho_{0}\right)$ is considered as variational parameter according to the Bethe method.

Now, the Hamiltonian $\left(\hat{H}^{(e, h)}\right)$ of uncoupling electron and hole is written as

$\widehat{H}^{(e, h)}=\widehat{H}_{0}^{(e, h)}+\Delta \widehat{\mathrm{H}}^{(e, h)}$,

where

$$
\begin{aligned}
& \hat{H}_{0}^{(e, h)}=-\frac{\hbar^{2}}{2}\left(\vec{\nabla}_{\rho, \varphi}^{(e, h)} \frac{1}{\mu^{(e, h)}(\rho)} \vec{\nabla}_{\rho, \varphi}^{(e, h)}+\right. \\
& \left.+\frac{1}{\mu^{(e, h)}(\rho)} \frac{\partial^{2}}{\partial z^{2}}\right)+U^{(e, h)}(\rho)
\end{aligned}
$$

is the main part of the Hamiltonian describing the electron and hole with effective masses $\mu^{(e, h)}(\rho)$ and potential energies $U^{(e, h)}(\rho)$ in the multi-shell cylindrical nanostructure.

The correction accounting the difference between the exact $\left(\hat{H}^{(e, h)}\right)$ and approximating $\left(\hat{H}_{0}^{(e, h)}\right)$ Hamiltonians

$$
\begin{aligned}
& \Delta \hat{H}^{(e, h)}=U^{(e, h)}(\rho, \varphi)-U^{(e, h)}(\rho)+ \\
& +\frac{\hbar^{2}}{2}\left[\vec{\nabla}_{\rho, \varphi}\left(\frac{1}{\mu^{(e, h)}(\rho)}-\frac{1}{\mu^{(e, h)}(\rho, \varphi)}\right) \vec{\nabla}_{\rho, \varphi}-\right. \\
& \left.-\left(\frac{1}{\mu^{(e, h)}(\rho)}-\frac{1}{\mu^{(e, h)}(\rho, \varphi)}\right) \frac{\partial^{2}}{\partial z^{2}}\right]
\end{aligned}
$$

is further considered as perturbation.

The Schroedinger equation with Hamiltonian (5) is solved exactly. The electron and hole wave functions (indices $e, h$ are not temporarily used) are taken as: 
$\Psi_{m k}(\vec{r})=\frac{1}{\sqrt{2 \pi L}} R_{m k}(\rho) e^{i k z} e^{i m \varphi}$

with the radial functions

$$
R_{m k}(\rho)= \begin{cases}R_{m k}^{(0)}=A_{m}^{(0)} J_{m}\left(k_{0} \rho\right), & 0 \leq \rho \leq \rho_{0} \\ R_{m k}^{(1)}=A_{m}^{(1)} I_{m}\left(k_{1} \rho\right)+B_{m}^{(1)} K_{m}\left(k_{1} \rho\right), & \rho_{0} \leq \rho \leq \rho_{1} \\ R_{m k}^{(2)}=A_{m}^{(2)} J_{m}\left(k_{0} \rho\right)+B_{m}^{(2)} N_{m}\left(k_{0} \rho\right), & \rho_{1} \leq \rho \leq \rho_{2} \\ R_{m k}^{(3)}=B_{m}^{(3)} K_{m}\left(k_{1} \rho\right), & \rho \geq \rho_{2}\end{cases}
$$

Here,

$k_{0}=\sqrt{2 \mu_{0} E / \hbar^{2}-k^{2}}, k_{1}=\sqrt{2 \mu_{1}\left(U_{0}-E\right) / \hbar^{2}+k^{2}}$,

$k$ is the axial quasi-momentum; $m=0, \pm 1, \pm 2, \ldots$ magnetic quantum number; $L$ - effective region for the electron movement along the nanostructure axis; $J_{m}, N_{m}$ - Bessel functions of the whole order, $I_{m}, K_{m}$ - modified Bessel functions.

The conditions for wave functions and their densities of currents continuity at three nanostructure interfaces $\left(\rho=\rho_{0}, \rho_{1}, \rho_{2}\right)$

$$
\left\{\begin{array}{l}
R_{m k}^{(i)}\left(\rho_{i}\right)=R_{m k}^{(i+1)}\left(\rho_{i}\right) \\
\left.\frac{1}{\mu_{i}} \frac{\partial R_{m k}^{(i)}(\rho)}{\partial \rho}\right|_{\rho=\rho}=\left.\frac{1}{\mu_{i+1}} \frac{\partial R_{m k}^{(i+1)}(\rho)}{\partial \rho}\right|_{\rho=\rho} \quad(i=0,1,2)
\end{array}\right.
$$

together with normalizing condition

$$
\int_{0}^{\infty}\left|R_{m k}(\rho)\right|^{2} \rho d \rho=1
$$

determine all the unknown coefficients: $A_{m}^{(i)}, B_{m}^{(i)}(i=$ $0,1,2,3)$ as well as analytical expressions for $\Psi_{n_{\rho} m k}^{(0)}\left(\rho_{0}, \vec{r}\right)$ wave functions and dispersion equation. Their solutions are numbered by the radial quantum number: $n_{\rho}=1,2 \ldots$ and define the electron (hole) energy spectrum $\left(E_{n_{\rho} m}^{(0)}\left(\rho_{0}, k\right)\right)$ as a function of $\rho_{0}$ being the variational parameter in zeroth approximation of the perturbation method.

Further, according to the Bethe method, we calculate the corrections of the first order to the energies of both quasi-particles $(e, h)$ as functions of $\rho_{0}$

$$
\begin{aligned}
& \Delta E_{n_{\rho} m}\left(\rho_{0}, k\right)= \\
& =6 \int_{-L / 2}^{L / 2} \sum_{j=0}^{2} \int_{S_{j}} \Psi_{n_{\rho} m k}^{(0) *}\left(\rho_{0} ; \rho, \varphi, z\right) \Delta \hat{H} \Psi_{n_{\rho} m k}^{(0)}\left(\rho_{0} ; \rho, \varphi, z\right) d \rho d \varphi d z .
\end{aligned}
$$

We must note that, due to the evident analytical properties of the perturbation Hamiltonian (6), the integration over $\rho, \varphi$ variables in expression (12) is performed only over $S_{j}$ regions, situated between the respective hexagons and approximating circles (toned regions in Fig. 1).

Now, the energy spectra of uncoupling electron and hole, as functions of variational parameter and axial quasi-momentum, are already known in the first approximation of perturbation method

$$
E_{n_{\rho} m}\left(\rho_{0}, k\right)=E_{n_{\rho} m}^{(0)}\left(\rho_{0}, k\right)+\Delta E_{n_{\rho} m}\left(\rho_{0}, k\right) .
$$

In order to study the exciton states in the multishell hexagonal nanostructure, one has to solve the stationary Schroedinger equation. Its Hamiltonian, besides the energy gap of quantum wire and nanotube material, Hamiltonians of uncoupling electron and hole, contains the energy of their interaction $\left(U\left(\left|\vec{r}_{e}-\vec{r}_{h}\right|\right)=-e^{2} /\left(\varepsilon\left(\vec{r}_{e}, \vec{r}_{h}\right)\left|\vec{r}_{e}-\vec{r}_{h}\right|\right)\right)$. The dielectric constant $\left(\varepsilon\left(\vec{r}_{e}, \vec{r}_{h}\right)\right)$ has a complicated dependence on the spatial location of quasi-particles in nanostructure.

Such problem can be solved within the method developed in details in refs. [6-8]. But the exciton binding energy obtained in the cited papers has the order of $10 \mathrm{meV}$, which is hundreds times smaller than the electron and hole energy of size quantization. The shifts of exciton energies due to the interaction with phonons are approximately $10 \mathrm{meV}$ [7] and do not essentially influence at the obtained results. Therefore, we do not take into account the electron-hole interaction and that of both quasi-particles with phonons.

Thus, the exciton energy spectrum $\left(E_{n_{\rho}^{h} m^{h}}^{n_{\rho}^{e} m^{e}}\right)$ at $k^{e}, k^{h}=0$, without considering the electron-hole and both quasi-particles interaction with phonons, is obtained from the condition of functional minimum

$$
\begin{aligned}
& E_{n_{\rho}^{h} m^{h}}^{n_{\rho}^{e} m^{e}}=E_{g_{0}}+\min \left(E_{n_{\rho}^{e} m^{e}}^{(0)}\left(\rho_{0}\right)+\Delta E_{n_{\rho}^{e} m^{e}}\left(\rho_{0}\right)\right. \\
& \left.+E_{n_{\rho}^{h} m^{h}}^{(0)}\left(\rho_{0}\right)+\Delta E_{n_{\rho}^{h} m^{h}}\left(\rho_{0}\right)\right\}_{\rho_{0}=\bar{\rho}_{0}},
\end{aligned}
$$

realized at $\rho_{0}=\bar{\rho}_{0}$. The same magnitude $\left(\bar{\rho}_{0}\right)$ determines the wave functions of exciton moving in the plane perpendicular to the nanostructure axis

$$
\begin{aligned}
& \left.\right|_{n_{\rho}^{h} m^{h}} ^{n_{\rho}^{e} m^{e}}>=\Psi_{n_{\rho}^{h} m^{h}}^{n_{\rho}^{e} m^{e}}\left(\bar{\rho}_{0} ; \rho_{e}, \varphi_{e}, \rho_{h}, \varphi_{h}\right)= \\
& =\Psi_{n_{\rho}^{e} m^{e}}\left(\bar{\rho}_{0} ; \vec{\rho}_{e}\right) \Psi_{n_{\rho}^{h} m^{h}}\left(\bar{\rho}_{0} ; \vec{\rho}_{h}\right) .
\end{aligned}
$$

Using the obtained wave functions, we can evaluate the intensities of inter-band optical quantum transitions [9]

$$
I_{n_{\rho}^{h} m^{h}}^{n_{\rho}^{e} m^{e}} \sim\left|\int_{0}^{\infty} R_{n_{\rho}^{e} m^{e}}(\rho) R_{n_{\rho}^{h} m^{h}}(\rho) \rho d \rho\right|^{2} .
$$


The numeric calculation of exciton energies and intensities of inter-band quantum transitions at $k^{e}, k^{h}=0$ is performed for $\mathrm{GaAs} / \mathrm{Al}_{0.4} \mathrm{Ga}_{0.6} \mathrm{As}$ hexagonal nanostructure experimentally grown in [5].

\section{Discussion of results}

Evolution of exciton spectrum as a function of nanostructure geometrical parameters is studied for $\mathrm{GaAs} / \mathrm{Al}_{0.4} \mathrm{Ga}_{0.6} \mathrm{As}$ multi-shell hexagonal nanotube with the following physical characteristics $[4,5,10]$ : $\mu_{0}^{e}=0.063 m_{0}, \quad \mu_{1}^{e}=0.096 m_{0}, \quad \mu_{0}^{h}=0.51 m_{0}$, $\mu_{1}^{h}=0.61 m_{0}, \quad U_{0}^{e}=297 \mathrm{meV}, \quad U_{0}^{h}=224 \mathrm{meV}$, $E_{g 0}=1520 \mathrm{meV}\left(m_{0}-\right.$ pure electron mass in vacuum); $a_{\mathrm{GaAs}}=5.65 \AA-$ GaAs lattice constant.

In Fig. 2, the energies of electron $E_{n_{\rho} 0}^{e}$ (a), exciton $E_{n_{\rho} 0}^{n_{\rho}^{e} 0}(\mathrm{~b})$, heavy hole $E_{n_{\rho} 0}^{h}$ (c) and intensities of

a)

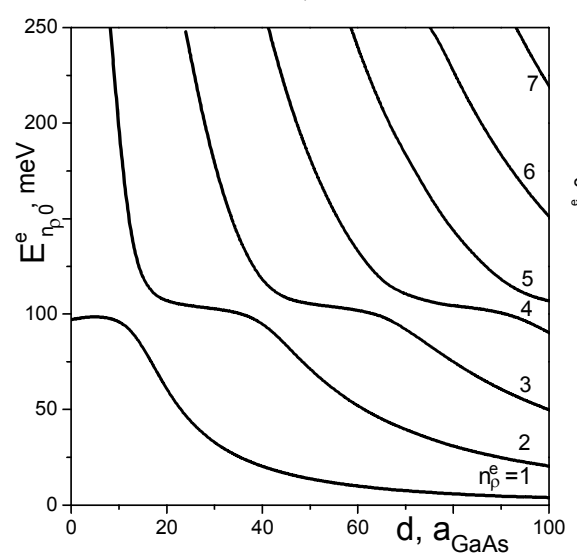

c)

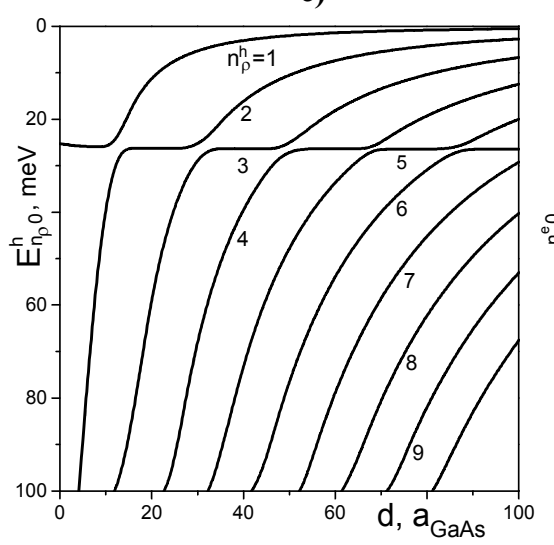

interband quantum transitions $I_{n_{\rho}^{h} 0}^{n_{\rho}^{e} 0}$ (d) are shown as functions of inner wire ( GaAs) diameter $(d)$ for the experimentally obtained magnitudes of barrier-shell ( $\Delta=4 \mathrm{~nm})$ and nanotube $(h=4 \mathrm{~nm})$ widths [5].

In Figs. 2a and 2c, one can see a finite number of energy levels at $d=0$. These levels coincide with those for the hexagonal nanotube (GaAs) embedded into $\mathrm{Al}_{0.4} \mathrm{Ga}_{0.6} \mathrm{As}$, which is proven by physical considerations.

The new electron and hole energy levels arise when the quantum wire appears and its diameter $(d)$ increases. The whole spectra shift into the region of smaller energies and the anti-crossings of energy levels are observed. The anti-crossing phenomenon is caused by the splitting of energy levels due to the tunnel effect present between the quantum wire (with the diameter $d$ ) and nanotube (with the width $h$ ) through the finite potential barrier (with the width $\Delta$ ). Both the electron and hole are located in the space of nanotube at that b)

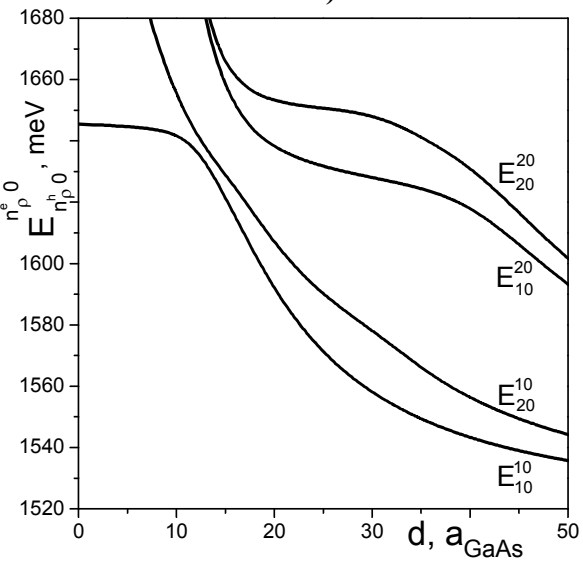

d)

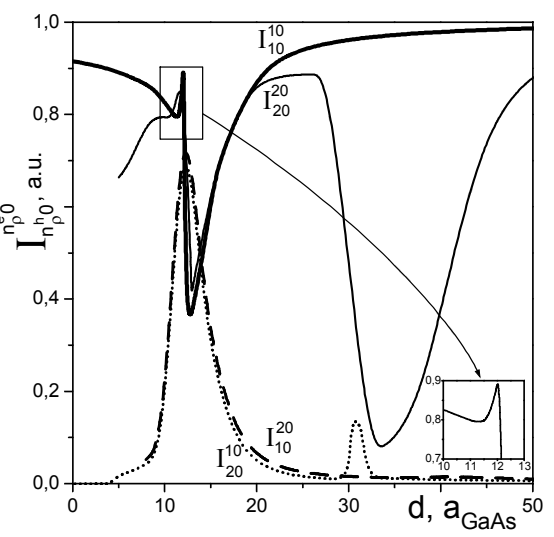

e)

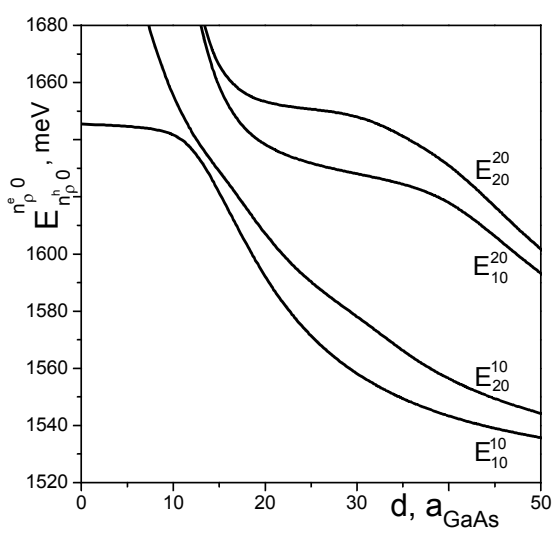

Fig. 2. Dependences of electron energy $E_{n_{\rho} 0}^{e}(\mathrm{a})$, exciton energy $E_{n_{\rho} 0}^{n_{\rho}^{e} 0}(\mathrm{~b})$, heavy hole energy $E_{n_{\rho} 0}^{h}(\mathrm{c})$, and intensities of interband quantum transitions $I_{n_{\rho}^{h} 0}^{n_{\rho}^{e} 0}$ (d) on the inner wire diameter $(d)$ at experimentally obtained magnitudes of barrier-shell width $(\Delta=$ $4 \mathrm{~nm})$ and nanotube width $(h=4 \mathrm{~nm})[5]$. 
a)

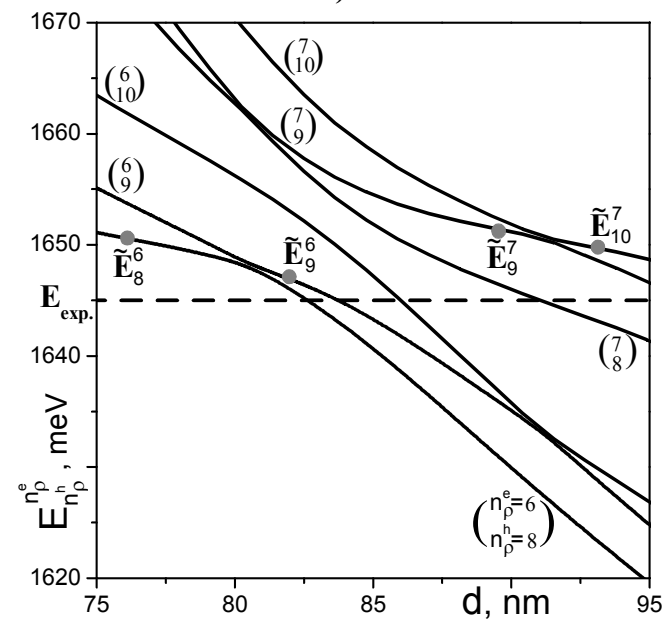

b)

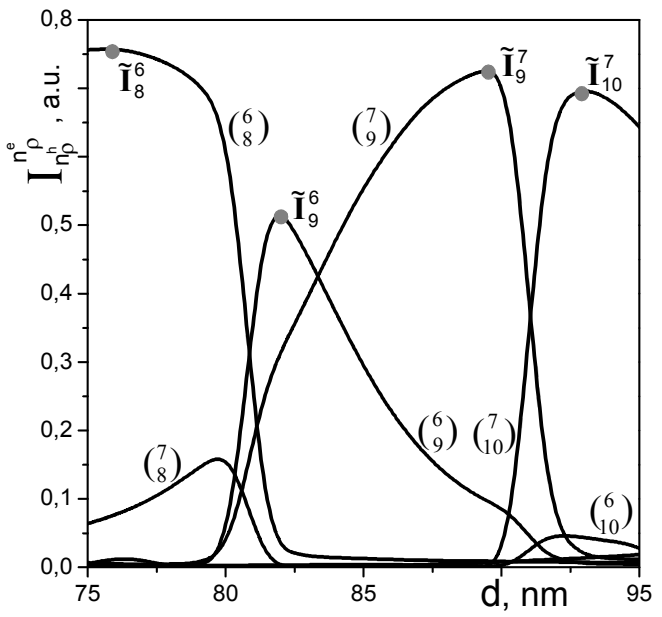

Fig. 3. Dependences of exciton energies $E_{n_{\rho}^{h}}^{n_{\rho}^{e}}$ (a) and intensities of interband quantum transitions $I_{n_{\rho}^{h}}^{n_{\rho}^{e}}$ (b) on the

plots of $E_{n_{p} 0}^{(e, h)}$ dependences on $d$, where the energies of quasi-particles are almost not changed. The plots where the energies of both quasi-particles decrease rapidly, correspond to the states in which the electron and hole are located in inner wire with the probability close to unity.

Evolution of exciton energies $\left(E_{n_{\rho}^{h} 0}^{n_{\rho}^{e} 0}\right)$ as functions of the inner wire diameter $(d)$, Fig. $2 \mathrm{~b}$, is evidently caused by the peculiarities of electron and hole spectra. In particular, the anti-crossing of exciton energy levels is observed as manifestation of electron and hole energy levels anti-crossings.

Fig. $2 \mathrm{~d}$ proves that the intensities of quantum transitions $\left(I_{n_{\rho}^{h} 0}^{n_{\rho}^{e} 0}\right)$ for all states non-monotonously depend on the inner wire diameter $(d)$, approaching several minimal and maximal magnitudes. Such $I_{n_{\rho}^{h} 0}^{n_{\rho}^{e} 0}$ behaviour is quite clear from the physical viewpoint. Really, when the electron and hole are in their ground states, the intensity of this transition $\left(I_{10}^{10}\right)$ at $d=0$ is close to unity ( $I_{10}^{10} \approx 0.9$ ). Then, both quasi-particles are located in the space of nanotube (with the width $h=$ $4 \mathrm{~nm}$ ), and overlapping of their wave functions is essential. When the inner wire appears and its diameter increases, the intensity decreases because the wave function of electron as "light" quasi-particle, more and more worms into the inner wire, while the heavy hole does not change its location in the nanostructure. For some critical $d$ magnitude, the heavy hole skip-like changes its location from nanotube into the inner wire. Herein, the intensity of transition first increases (see the insert in Fig. 2d) and then rapidly reduces. The minimal magnitude of intensity ( $I_{10}^{10} \approx 0.35$ ) is obtained when the hole with the probability close to unity is located in the inner wire, and the electron with equal probability $(\sim 0.5)$ is located in the inner wire and nanotube. The further intensity increase is observed when the electron and hole are located in the inner wire, and overlap of their wave functions increases for higher $d$ magnitudes.

The non-monotonous characters of intensities of interband quantum transitions between arbitrary states are explained in the analogous manner due to the changed location of both quasi-particles in the space of quantum wire and nanotube.

In order to compare the theoretical and experimental data, the exciton energies ( $E_{n_{\rho}^{h}}^{n_{\rho}^{e}}$, Fig. 3a) and respective intensities of quantum transitions ( $I_{n_{\rho}^{h}}^{n_{\rho}^{e}}$, Fig. 3b) are presented as functions of the inner wire diameter $(d)$ within the range: $d=85 \pm 10 \mathrm{~nm}$. It corresponds to the sizes of nanotube, for which the luminescence peak (at $E_{\text {exp }} \approx 1645 \mathrm{meV}$ ) was experimentally observed [5]. The numeric calculations of exciton energies and intensities of quantum transitions are performed for magnetic quantum number equal to zero, thus index " 0 " is not used in the figure for convenience.

Both figures prove that only four out of six exciton energy curves: $E_{8}^{6}, E_{9}^{6}, E_{9}^{7}, E_{10}^{7}$ can be characterized by intensities higher than 0.5 . The exciton energies $\left(\widetilde{E}_{8}^{6}\right.$, $\left.\widetilde{E}_{9}^{6}, \widetilde{E}_{9}^{7}, \widetilde{E}_{10}^{7}\right)$, at which the luminescence peak must be experimentally observed, correspond to the intensity maxima $(\tilde{I})$. Thus, the luminescence peak, described in [5], can be produced by different exciton states with 
weakly differing energies, depending on an exact experimental diameter $(d)$ of the inner wire.

Finally, the calculations of probability density of electron and hole location $\left|R_{n_{\rho} m}^{(e, h)}(\rho)\right|^{2} \rho$ in nanostructure prove that both quasi-particles producing the exciton in the above mentioned states $\left(\widetilde{E}_{n_{\rho}^{h}}^{n_{\rho}^{e}}\right)$ are localized in nanotube with the width $h$.

\section{Conclusions}

1. The theory of exciton spectra and intensities of quantum transitions for the multi-shell hexagonal nanostructure has been developed by applying the models of effective masses and rectangular potentials for the electron and hole by using the Bethe variational method.

2. It has been shown that the intensities of interband quantum transitions for all the states nonmonotonously depend on inner wire diameter $(d)$ approaching several minimal and maximal magnitudes. Evolution of intensities is quite explained by the complicated character of distribution of probability density of electron and hole location in the space of multi-shell nanostructure.

3. It has been established that the numeric results for exciton energy spectrum and intensities of quantum transitions, obtained within the developed theory, well correlate with the experimental data for the radiation spectrum of nanotubes grown using $\mathrm{GaAs} / \mathrm{Al}_{\mathrm{x}} \mathrm{Ga}_{1-\mathrm{x}} \mathrm{As}$ semiconductors.

\section{References}

1. A.I. Persson, M.W. Larsson, S. Stenstro, B.J. Ohlsson, L. Samuelson, L.R. Wallenberg, Solid-phase diffusion mechanism for GaAs nanowire growth // Nat. Mater. 3(10), p. 677-681 (2004).
2. P. Mohan, J. Motohisa, T. Fukui, Realization of conductive InAs nanotubes based on latticemismatched InP/InAs core-shell nanowires // Appl.Phys. Lett. 88(1), 013110 (2006).

3. P. Mohan, J. Motohisa, T. Fukui, Fabrication of $\mathrm{InP} / \mathrm{InAs} / \mathrm{InP} \quad$ core-multishell heterostructure nanowires by selective area metalorganic vapor phase epitaxy // Appl. Phys. Lett. 88(13), 133105 (2006).

4. M. Heigoldt, J. Arbiol, D Spirkoska, J.M. Rebled
S. Conesa-Boj,
G. Abstreiter,
F. Peiro, J. R. Morantece, A. Fontcuberta i Morral, Long range epitaxial growth of prismatic heterostructures on the facets of catalyst-free GaAs nanowires // J. Mater. Chem. 19(7), p. 840-848 (2009).

5. A. Fontcuberta i Morral, D. Spirkoska, J. Arbiol, M. Heigoldt, J. R. Morante, G. Abstreiter, Prismatic quantum heterostructures synthesized on molecular-beam epitaxy GaAs nanowires // Small, 4(7), p. 899-903 (2008).

6. N. Tkach, A. Makhanets, N. Dovganiuk, Theory of the exciton spectrum of an array of widely spaced hexagonal nanotube // Physics of the Solid State, 51(12), pp. 2529-2536 (2009).

7. M. Tkach, O. Makhanets, M. Dovganiuk, O. Voitsekhivska, Exciton spectrum in hexagon nanotube accounting exciton-phonon interaction // Physica E, 41(8), p. 1469-1474 (2009).

8. M. Tkach, O. Makhanets, A. Gryschyk, R. Fartushynsky, Exciton in quantum tube with hexagon cross // Rom. J. Phys., 54(1-2), p. 37-45 (2009).

9. J.H. Davies, The Physics of Low-dimensional Semiconductors: An Introduction. Cambridge University Press, New York, p. 438 (1998).

10. G.Q. Hai, F.M. Peeters, J.T. Devreese, Electron optical-phonon coupling in $\mathrm{GaAs} / \mathrm{Al}_{\mathrm{x}} \mathrm{Ga}_{1-\mathrm{x}} \mathrm{As}$ quantum wells due to interface, slab, and half-space modes // Phys. Rev. B., 48(7), p. 4666-4668 (1993). 